\title{
Thin Layer Chromatography and Chemometric Studies of Selected Potentilla Species
}

\author{
Ryszard Świeboda, Agnieszka Jóźwiak, Grzegorz Jóźwiak, Monika Waksmundzka-Hajnos \\ Department of Inorganic Chemistry, Medical University, Lublin, Poland \\ Email: monika.hajnos@umlub.pl
}

Received 13 September 2014; revised 28 October 2014; accepted 13 November 2014

Copyright (C) 2014 by authors and Scientific Research Publishing Inc.

This work is licensed under the Creative Commons Attribution International License (CC BY). http://creativecommons.org/licenses/by/4.0/

(c) (i) Open Access

\begin{abstract}
Herbal medicinal products have a long-standing therapeutic record. To ensure the quality of herbal products specific identification tests which allow experts to discriminate related species and/or potential adulterants/substitutes are required. The purpose of the research was to recommend an original and simple method for the separation of closely related five triterpenic acids (ursolic, oleanolic, tormentic, euscaphic, pomolic acid) and its application to chemotaxonomy studies. 17 standard samples of Potentilla species and 3 test samples were chromatographed (with or without prechromatographic derivatization) on silica gel plates using the mobile phase: chloroform-diethyl ether-methanol-formic acid (30:10:1:0.2 $\mathrm{v} / \mathrm{v} / \mathrm{v} / \mathrm{v})$; they were subsequently derivatized, and visualized in UV $366 \mathrm{~nm}$ light. After images received pre-treatment (montaging stack, removal of noise, background subtract, horizontal equalization, two types of warping) exploratory analysis of the investigated Potentilla species fingerprints was processed. The method without prechromatographic derivatization was used to create differential fingerprints. Good results were obtained by the application of r-project iterative algorithm for subtract background and our simple algorithm for warping to image processing of TLC chromatograms for the first time.
\end{abstract}

\section{Keywords}

Pomolic Acid, Ursolic Acid, Euscaphic Acid, Tormentic Acid, Oleanolic Acid, Triterpene Acids, TLC Fingerprint, Potentilla

\section{Introduction}

Herbal medicinal products have a long-standing therapeutic record and are still used as cure for various diseases

How to cite this paper: Świeboda, R., Jóźwiak, A., Jóźwiak, G. and Waksmundzka-Hajnos, M. (2014) Thin Layer Chromatography and Chemometric Studies of Selected Potentilla Species. American Journal of Analytical Chemistry, 5, 1109-1120. http://dx.doi.org/10.4236/ajac.2014.516118 
all over the world. It is the quality of herbal medicinal products that is of major importance since chemical complexity of herbs varies greatly due to a wide range of factors such as species variation, hybrids, growth location and condition, climate, harvesting season and condition as well as drying and storage condition. To ensure the quality of herbal medicinal products, European Medicines Agency (EMA) suggest establishing good agricultural and collection practice for herbal starting materials (GACP) [1] and emphasize specific identification testing which allows experts to discriminate related species and/or potential adulterants/substitutes [2]. Herbal fingerprinting was adopted as a comprehensive qualitative methodology in which the entire chromatogram is assessed during data analysis and enables to distinguish different species occurring within the same herbal family [3]. By definition, the chromatographic fingerprint of herbal medicinal products is, in practice, the chromatographic pattern of the extract of some common chemical components of pharmacologically active and or chemically characteristics [4]. Thin layer chromatography (TLC) is frequently used as a chromatographic technique in fingerprint analysis of herbal medicines. The advantages of TLC method in the construction of such fingerprints are its simplicity, versatility, high velocity, specific sensitivity and simple sample preparation. Therefore, TLC is considered to be a very convenient method in quality determination and in tracing possible adulteration of herbal products [4].

The aim of this paper is to elaborate TLC method for the separation five triterpene acids (ursolic acid (UA), oleanolic acid (OA), pomolic acid (PA), tormentic acid (TA), euscaphic acid (EA)) present in the Potentilla species. The method was applied to construct the fingerprints of dichloromethane extracts of roots and rhizomes in the Potentilla species. This type of secondary metabolites was chosen on purpose because of its wide spectrum of pharmacological activities. Triterpene acids are reported to have an interesting bioactivity, including anti-HIV [5], hepatoprotective [6] [7], antimalarial [8], antimicrobial and anti-inflammatory effect [9], anti-tumoral activity [10] [11], anti-atherogenic [12] and hypoglycemic effect [13].

The separation of triterpene acids by thin layer chromatography was carried out using selected methods. The research found in recent papers points to simultaneous separation of various triterpene acids such as: oleanolic acid, $\beta$-amyrin and $\beta$-amyrin acetate on silica gel HPTLC plates using hexane-dichloromethane-methanol-water (4:5:0.9:0.1) [14], oleanolic and ursolic acid on silica gel HPTLC plates using petroleum ether-ethyl acetateacetone (8.2:1.8:0.1) after pre-chromatographic iodine derivatization [15], ursolic acid and oleanolic acid and 8 other triterpenoids and sterols ( $\alpha$-amyrin, $\beta$-amyrin, $\delta$-amyrin, lupeol, lupenon, lupeol acetate, cycloartenol, cycloartenol acetate, stigmasterol and $\beta$-sitosterol) on C18 RP-HPTLC plates using acetone-acetonitrile (5:1) or ethyl acetate-acetonitrile (3:2) and on silica gel HPTLC plates using n-hexane-ethyl acetate (5:1) [16], oleanolic, ursolic and betulinic acids on C18 RP-HPTLC plates using n-hexane-ethyl acetate (5:1) after pre-chromatographic bromine derivatization [17].

The aim of this paper is to elaborate TLC separation method of pomolic, ursolic, oleanolic, euscaphic and tormentic acid and apply it to chemotaxonomy studies of various Potentilla species. The method as such hasn't been reported yet. The studies of this plant genus were carried out to find the specific method for identification of both species and subspecies in the Potentilla genus. TLC fingerprints (videoscans) of dichloromethane extracts obtained from roots and rhizomes of various Potentilla species were chemometrically processed with free open source software. Our current research shows new strategies for image processing and thorough analysis of fingerprints.

\section{Materials and Methods}

\subsection{Chemicals}

All chemicals including solvents were at least of analytical grade. Dichloromethane, chloroform, methanol, ethanol, acetone, diethyl ether, formic acid, iodine crystals, sulphuric acid ( $\geq 95 \%)$ were purchased from Polish Chemical Reagents (POCh, Gliwice, Poland).

The standard of ursolic acid (UA) ( $\geq 98.5 \%$ ), oleanolic acid (OA) (98.7\%), stigmasterol (96.5\%) were from Sigma-Aldrich (St. Louis, MO, USA), tormentic acid (TA) (98\%), euscaphic acid (EA) (95\%) and pomolic acid (PA) (98\%) were from LGC Standards (BioBioPha Co, Ltd., China). $\beta$-sitosterol (97.8\%) was from LGC Standards (Chromadex, USA). Chemical structures of triterpene acids are presented in Figure 1.

The plates were developed in horizontal Teflon DS chambers (Chromdes, Lublin, Poland). A rotary evaporator (Laborata 4003, Heidolph) was used for extracts evaporation. The ultrasonic bath Elmasonic S30H (Elma GmbH\&Co. KG, Singen, Germany) was used for the extraction of compounds from Potentilla species. 


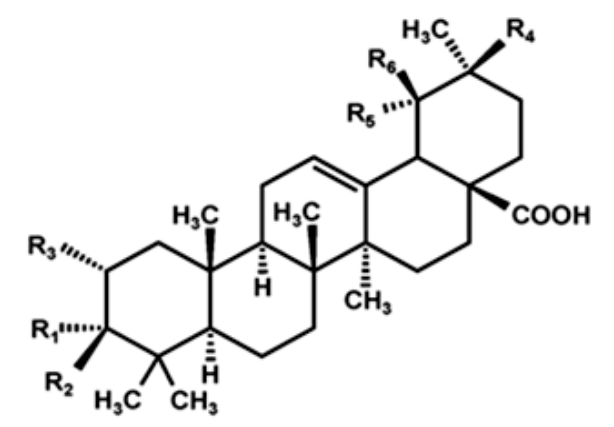

\begin{tabular}{|l|c|c|c|c|c|c|}
\hline & $R_{1}$ & $R_{2}$ & $R_{3}$ & $R_{4}$ & $R_{5}$ & $R_{6}$ \\
\hline Ursolic acid & $H$ & $\mathrm{OH}$ & $\mathrm{H}$ & $\mathrm{H}$ & $\mathrm{H}$ & $\mathrm{CH}_{3}$ \\
Oleanolic acid & $\mathrm{H}$ & $\mathrm{OH}$ & $\mathrm{H}$ & $\mathrm{CH}_{3}$ & $\mathrm{H}$ & $\mathrm{H}$ \\
Pomolic acid & $H$ & $\mathrm{OH}$ & $\mathrm{H}$ & $\mathrm{H}$ & $\mathrm{OH}$ & $\mathrm{CH}_{3}$ \\
Tormentic acid & $H$ & $\mathrm{OH}$ & $\mathrm{OH}$ & $\mathrm{H}$ & $\mathrm{OH}$ & $\mathrm{CH}_{3}$ \\
Euscaphic acid & $\mathrm{OH}$ & $\mathrm{H}$ & $\mathrm{OH}$ & $\mathrm{H}$ & $\mathrm{OH}$ & $\mathrm{CH}_{3}$ \\
\hline
\end{tabular}

Figure 1. The structure of triterpene acids.

\subsection{Preparation of Standard Solutions}

Standard solutions of EA, PA, OA and UA were prepared by dissolving $1 \mathrm{mg}$ of each compound in $1 \mathrm{~mL}$ of acetone. Standard solution of TA were prepared by dissolving $0.5 \mathrm{mg}$ in $1 \mathrm{~mL}$ of acetone. All standard solutions were stored at $4^{\circ} \mathrm{C}$.

\subsection{Sample Preparation}

Plant material was collected in Maria Curie-Sklodowska University Botanical Garden, Lublin, Poland. Roots and rhizomes of the Potentilla species were examined and treated as standard samples (see Table 1). Three test samples collected by authors in different parts of Europe and in different time (the Bialystok Region-Poland, harvest year: 2008, the Zamosc Region-Poland, harvest year: 2010, central France, harvest year: 2011) were also examined.

Plant material was dried at room temperature for 10 days during which it was turned over every day and subsequently reduced to powder (mesh $0.315 \mathrm{~mm}$ ), using a microfine grinder drive IKA Werke MF 10 basic (Staufen, Germany), and stored at room temperature.

$3.00 \mathrm{~g}$ of dry powdered roots and rhizomes of the Potentilla species were extracted three times with $50 \mathrm{~mL}$ portions of dichloromethane within $30 \mathrm{~min}$ in an ultrasonic bath at the temperature of $30^{\circ} \mathrm{C}$. The extracts were combined and evaporated to dryness using rotary evaporator under reduced pressure. Dry residues were further dissolved in acetone to fixed volume of $10 \mathrm{~mL}$.

\subsection{Preparation of Dipping Detection Reagent}

$10 \%$ (v/v) $\mathrm{H}_{2} \mathrm{SO}_{4}$ in ethanol detection reagent was prepared by mixing concentrated sulphuric acid ( $\left.\geq 95 \%\right)$ (10 $\mathrm{mL}$ ) and ethanol $(90 \mathrm{~mL})$. The mixture was cooled in an ice bath.

\subsection{Prechromatographic Derivatization}

One plate (with P. megalantha and all standards), see Figure 2(b), was developed with $1 \%$ iodine solution in chloroform at the distance of $1.2 \mathrm{~cm}$. Next, the starting zone was covered by a glass strip and the plates were placed in darkness for $10 \mathrm{~min}$. When the reaction was completed, the plates were dried in the stream of warm air to remove the excess of iodine.

Due to the changes in the appearance of chromatograms after iodine derivatization (the appearance of new, non-typical spots or disappearance/weakening of the already existing ones) we decided to apply the method without prechromatographic derivatization for the construction of Potentilla species fingerprints. 
Table 1. Potentilla species.

\begin{tabular}{cc}
\hline Potentilla species & Collecting conditions \\
Potentilla anserina L. & Botanical Garden, Lublin, Poland, 2012 \\
Potentilla argyrophylla Wall. ex Lehm. & Botanical Garden, Lublin, Poland, 2012 \\
Potentilla atrosanguinea G. Lodd. ex D. Don & Botanical Garden, Lublin, Poland, 2012 \\
Potentilla bifurca L. & Botanical Garden, Lublin, Poland, 2012 \\
Potentilla collina Wibel & Botanical Garden, Lublin, Poland, 2012 \\
Potentilla crantzii (Crantz) Beck ex Fritsch & Botanical Garden, Lublin, Poland, 2012 \\
Potentilla desertorum Bunge & Botanical Garden, Lublin, Poland, 2012 \\
Potentilla erecta (L.) Raeusch & Botanical Garden, Lublin, Poland, 2012 \\
Potentilla fulgens Wall. ex Lehm. & Botanical Garden, Lublin, Poland, 2012 \\
Potentilla grandiflora L. & Botanical Garden, Lublin, Poland, 2012 \\
Potentilla hirta L. & Botanical Garden, Lublin, Poland, 2012 \\
Potentilla megalantha Takeda & Botanical Garden, Lublin, Poland, 2012 \\
Potentilla montenegrina Pant. & Botanical Garden, Lublin, Poland, 2012 \\
Potentilla neumanniana Rchb & Botanical Garden, Lublin, Poland, 2012 \\
Potentilla recta L. subsp. obscura (Willd.) Arcang. & Botanical Garden, Lublin, Poland, 2012 \\
Potentilla recta L. & Botanical Garden, Lublin, Poland, 2012 \\
Potentilla reptans L. & Botanical Garden, Lublin, Poland, 2012 \\
Test sample 1 & Bialystok Region, Poland, 2008 \\
Test sample 2 & Central France, 2011 \\
Test sample 3 & Zamosc Region, Poland, 2010 \\
\hline
\end{tabular}
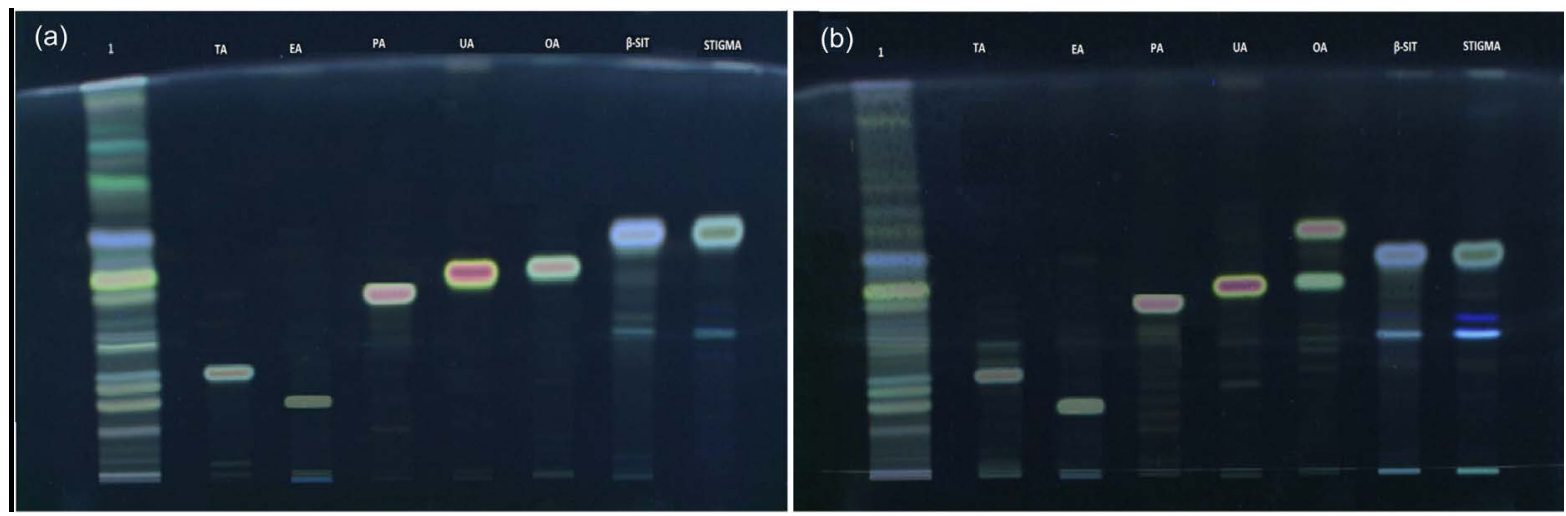

Figure 2. TLC silica gel $60 \mathrm{~F}_{254}$ developed in Teflon DS chamber using chloroform-diethyl ether-methanol-formic acid (30:10:1:0.2 v/v) after derivatization with $10 \% \mathrm{H}_{2} \mathrm{SO}_{4}$ in ethanol detection reagent in UV $366 \mathrm{~nm}$ light. (a) Without prechromatographic derivatization; (b) With prechromatographic derivatization with $1 \%$ iodine solution in chloroform; Tracks: 1-P. megalantha, tormentic acid (TA), euscaphic acid (EA), pomolic acid (PA), ursolic acid (UA), oleanolic acid (OA), $\beta$-sitosterol ( $\beta$-SIT), stigmasterol (STIGMA).

\subsection{Thin-Layer Chromatography}

TLC was performed on $10 \mathrm{~cm} \times 20 \mathrm{~cm}$ plates coated with silica gel $60 \mathrm{~F}_{254}$ Art. No. 1.05554 (Merck, Darmstadt, Germany). $10 \mu \mathrm{L}$ of standard solutions of ursolic acid, oleanolic acid, tormentic acid, euscaphic acid, pomolic acid, $\beta$-sitosterol, stigmasterol and $10 \mu \mathrm{L}$ of herbal extracts were spotted on the plates. To construct fingerprint, twenty samples of Potentilla extracts were applied on four plates. The same species or subspecies were on the same plate (tracks of the first plate: 1 , P. anserina; 2, P. hirta; 3, P. fulgens; 4, P. crantzii; 5, P. grandiflora; tracks of the second plate: $6, P$. montenegrina; 7, P. recta subsp. obscura; 8 , P. recta; 9 , P. atrosanguinea; $10, P$. atrosanguinea; tracks of the third plate: 11 , P. erecta; 12 , P. desertorum; 13, P. collina; 14 , P. megalantha; $15, P$. bifurca; tracks of the fourth plate: 16, P. neumanniana; 17, P. reptans; 18, Bialystok Region, 2008; 19, France, 2011; 20, Zamosc Region, 2010). 
The plates were developed in horizontal Teflon DS chambers (Chromdes, Lublin, Poland) with a mixture of chloroform, diethyl ether, methanol, formic acid (30:10:1:0.2 v/v) as a mobile phase at the distance of $8 \mathrm{~cm}$.

Standards and sample solutions were applied as $15 \mathrm{~mm}$ bands, $20 \mathrm{~mm}$ from the left and right edge, $10 \mathrm{~mm}$ from the bottom edge and $35 \mathrm{~mm}$ apart by means of Linomat 5 (CAMAG, Muttenz, Switzerland), operated with win CATS (Planar Chromatography Manager) version 1.4.0. (CAMAG, Muttenz, Switzerland), and syringe $100 \mu \mathrm{l}$ (No. 695.0014) (Hamilton, Bonaduz, Switzerland).

After being dried in the stream of warm air the plates were sprayed with $10 \%(\mathrm{v} / \mathrm{v}) \mathrm{H}_{2} \mathrm{SO}_{4}$ in ethanol detection reagent by means of TLC sprayer GW1 108540 (Merck, Darmstadt, Germany), dried for 5 min and then heated at $105^{\circ} \mathrm{C}$ for 3 min by means of TLC plate heater III (CAMAG, Muttenz, Switzerland).

The documentation of chromatographic plates was obtained with using a TLC Visualizer CAMAG in white light and UV 366 nm light by means of DigiStore 2 Documentation System (CAMAG, Muttenz, Switzerland), operated with winCATS Version 1.4.1.8154 software. The plates were documented within $10 \mathrm{~min}$, afterwards progressive degradation was observed.

To ensure the repeatability of results we carried out the analysis on the same TLC plates (the same producer and batch lot). The samples were applied by means of Linomat 5 . The chromatograms were developed in horizontal Teflon DS chambers with a mobile phase prepared in the amount sufficient to develop all plates. The plates were sprayed by means of TLC sprayer with $10 \% \mathrm{H}_{2} \mathrm{SO}_{4}$ in ethanol detection reagent, prepared in the amount sufficient to spray all plates. The time of drying, heating as well as the time before taking a photo were strictly obeyed.

Such developed analytical method of the identification of five triterpenic acids was validated by the determination of specificity, repeatability and robustness.

The most common type of fingerprint analysis relied upon the data of methanol extracts analysis. Such extracts contain a number of compounds with different chemical structure, which facilitates the differentiation of the species of the same genus. In our study we used dichloromethane extracts which contained a relatively small group of compounds, which caused that the development of methods indicating the interspecies differences was much more difficult. The chemometric attempt shows that, for such difficult cases to develop, the method using image processing can be very effective.

\section{Results and Discussion}

\subsection{Data Pre-Treatment}

As mentioned above, for fingerprint construction four original TLC images with 5 samples of Potentilla extracts on each plate in UV $366 \mathrm{~nm}$ light were prepared. The original TLC images were exported from winCATs TLC workstation (CAMAG) to ImageJ (1.48c version), public domain, Java-based image processing program developed at the National Institute of Health. ImageJ was designed with open architecture that provides extensibility via Java plugins and recordable macros. From every track on the plate the subtracks was cut out (715 pixel length and 60 pixel width for each subtrack). Next the stack of all subtracks (as RGB color images) was montage. Three RGB channels were always processed separately, paralell. The stack of all subtracks was decomposed to three independent channels for red, green and blue primary color components before every step of image processing. The montaging stack should be a basic step in processing large number of images. For each stage of image processing special macro instruction was written. These executing algorithms were designed to automate all actions and make this process less tedious and less error-prone.

Subsequently, to increase the signal-to-noise ratio without greatly distorting the signal we used the SavitzkyGolay filter [18] which performs a simple local least squares regression and provides its fitted value to the output. The removal of noise was done using the Savitzky Golay Filter Plugin. This type of filter was previously performed for the removal of noise in Raman spectra [19], in densitometry [20] or TLC videoscans [21] TLC analysis is affected by noise (e.g. related to grainy nature of TLC plates, non-uniformity of the plate spraying, progressive degradation of spot color). Therefore, the increase the signal-to-noise ratio is required. As it proves previously [21] the charged coupled device (CCD) camera noise is almost uncorrelated (white one) and it can be treated as homoscedastic (almost no correlation of signal amplitude with noise amplitude is observed). For this reason, the optimal Savitzky-Golay parameters were selected without checking its autocorrelation. The half width of the filter (the length of the window) and the degree of the fitted polynomial (order of filter) were optimized. The difference between the surface plot of original TLC image and the surface plot of a smoothed ver- 
sion of TLC image depending on the parameters used is presented in Figure 3. Optimal parameters which still retain features of TLC image (without over smoothing) were 8:3 (half width of filter: order of filter).

After smoothing the digital data in stack we subtracted the background. The built-in roller ball algorithm to remove the background baseline is implemented in ImageJ. The background estimated in this algorithm depends on the ball diameter, which has to be larger than the peak or the spot width. We decided to estimate of baseline (to remove it) by using another algorithm, based on "fill peak algorithm" described in baseline r-project package (free software environment for statistical computing and graphics). In this method, local windows of buckets the minimum of the mean and the previous iteration is chosen as the new baseline. In these windows of buckets the minimum of the mean and the previous iteration is chosen as the new baseline. The width of local window and the number of iterations in suppression loop were optimalized. The line, which is created by this algorithm, is treated as the background estimate (see Figure 4(b)). The subtract background procedure is illustrated in Figure 4.

Subsequently, horizontal equalization for image defects removal was adopted. Each color of RGB subtracks was compressed (using compressed) to the size of 715 pixel length and 1 pixel width. Afterwards, the size of subtracks was adjusted to primary size $(715 \times 60)$ and in the next step red, green and blue stacks merged. The horizontal equalization allowed us to eliminate the image defects that occurred during TLC analysis (e.g. alignment of color spots, accidental dots, spots not aligned horizontally) or during the warping (e.g. narrowing of the image).

Therefore, the aim of the study was also the chemometric processing of 20 chromatograms (precisely 20 subtracks in stack). For this reason it is required to perform the alignment of chromatograms by warping. The warping was carried out using bUnwarpJ developed as an ImageJ plugin [22], previously used for TLC videoscans [23]. This is an algorithm for elastic and consistent image registration and it performs a simultaneous registration of two images, source image and target image (source image is elastically deformed in order to look as similar as it is possible to target image). The images like ours (RGB Color images) were converted to grayscale during the registration process. The resulting transformations were applied to the original color images.

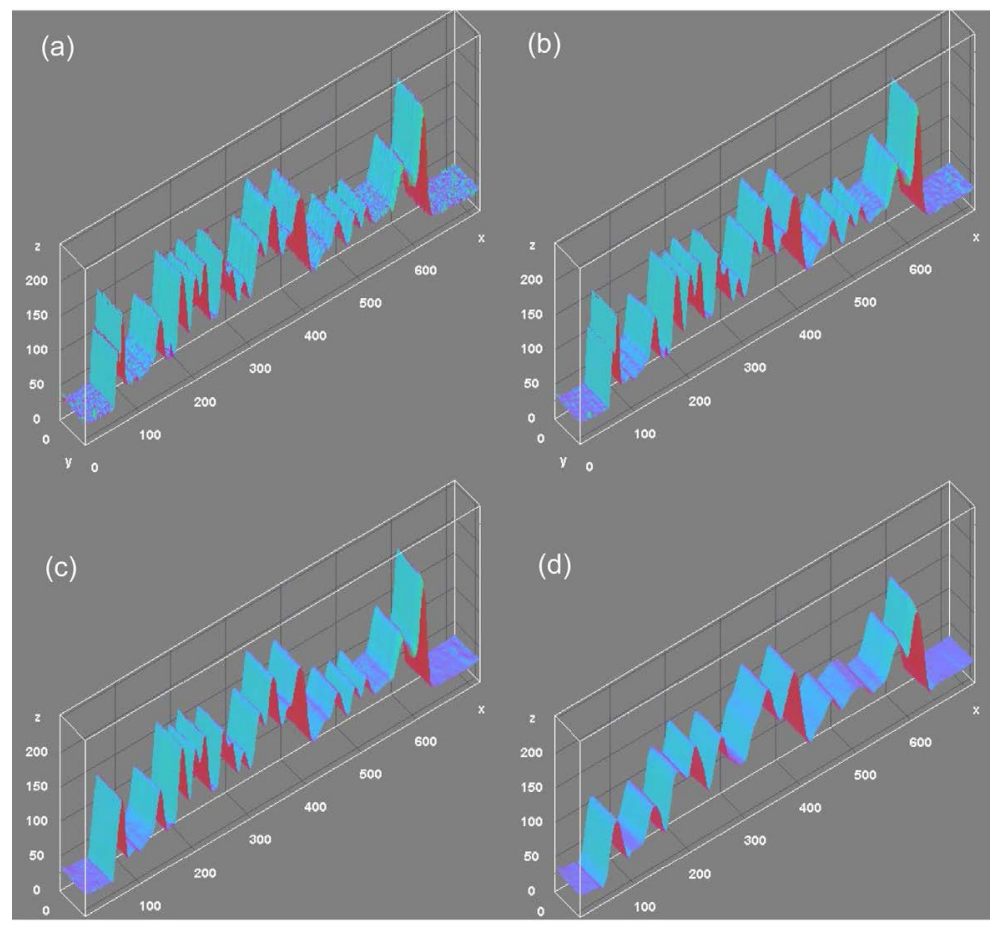

Figure 3. The Savitzky-Golay filter (half width of filter: order of filter). (a) Surface plot of original $P$. anserina TLC image; (b) Surface plot of a smoothed version $P$. anserina TLC image (6:4); (c) Surface plot of a smoothed version $P$. anserina TLC image (8:3); (d) (b) Surface plot of a smoothed version $P$. anserina TLC image (20:3). 

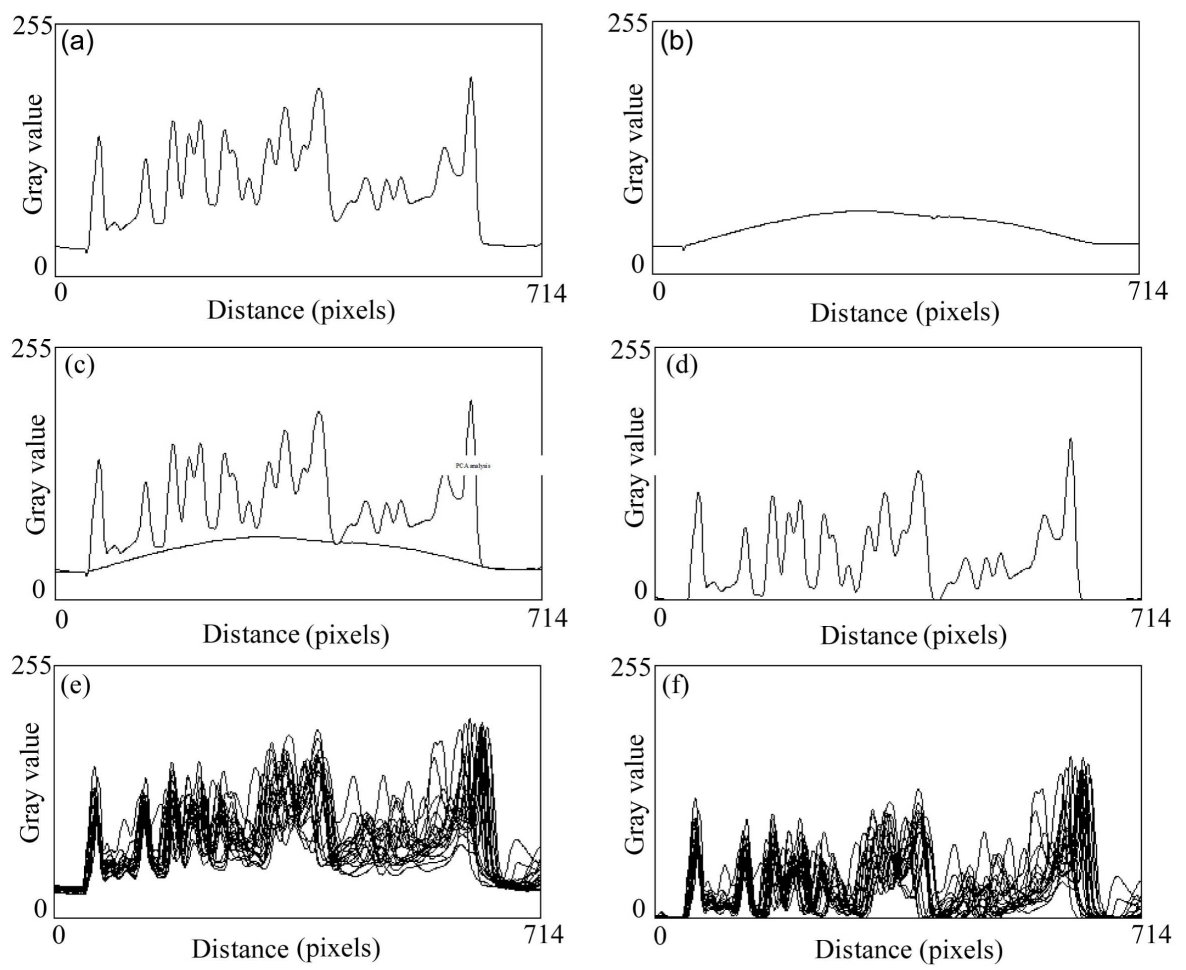

Figure 4. Subtract background: (a) P. anserina plot with background; (b) P. anserina background estimate plot; (c) P. anserina plot with background and background estimate; (d) $P$. anserina plot after subtract background; (e) Plot of all subtrack in stack; (f) Plot of all subtracks in stack after subtract background.

The first step of warping is the choice of target image. Following previously research [24], we selected the image with the highest mean correlation coefficient with remaining ones (in our research that was P. hirta chromatogram (subtrack 2) as the target image. The optimalization of image registering parameters was the second step. We selected the registration mode "Mono" which makes the program perform only unidirectional registration, i.e. from source image to target image. As it was observed, the great impact on the final result of warping seems to have three parameters: initial deformation option, sample subsample factor and curl weight. The initial deformation lists permit selecting the coarsest and finest scale of the spline deformation field (where "very coarse" option corresponds to 4 splines (one in each corner of the image). Sample subsample factor enables subsampling input images. Next registration was calculated using the subsampled versions of images. The results were applied to the original ones (e.g. image dimensions can be reduced by the factor between $0\left(2^{0}=1\right)$ and $\left.7\left(2^{7}=128\right)\right)$. We also observed that the warping result was different for the same stack, target image and that same warping parameters, but with different position of stack (vertical or horizontal) or with or without invert color before warping. The warping effect was then incorrect $\left(R_{f}\right.$ value of the spots identified as the same substance was changed) or with image defects (i.e. large image narrowing, arched spots). Only the use of markers significantly improved the quality of the process. The points being exactly in the middle of each image corresponding to the start line, finish line, and part of the spots of previously identified compounds (ursolic acid, $\beta$-sitostrol + stigmasterol) were selected as markers (landmarks).

To examine such characteristics, this observation prompted us to test TLC images to design our own simple algorithm for alignment. The algorithm was not based on B-spline but on segmental elastic compression or decompression of images between fixed markers. In addition to the landmarks previously used in warping, two points corresponding to the beginning and end of each subtrack were added. Because its simplicity the algorithm, didn't cause distortion of the image, and could be used practically without data smoothing, background subtract and horizontal equalization. This algorithm can be successfully used for similar TLC chromatograms, especially in cases where we have spots with confirmed identity preferably distributed in different parts of the chromatogram. 
Warping procedures are illustrated in Figure 5 and Figure 6. The comparison of two warping algorithms applied can be seen in the P. desertorum chromatogram example in Figure 6(b) (black plot—before warping, pink plot—after bUnwarpJ warping, blue plot—after our algorithm warping). Mean Pearson's correlation coefficient of all chromatograms (subtracks) increased from 0.582 before warping to 0.844 after bUnwarpJ warping, and to 0.859 after our algorithm warping.

\subsection{Exploratory Analysis of the Investigated Potentilla Species Fingerprints}

After data pre-treatment of fingerprints (removal of noise, subtract background, horizontal equalization,

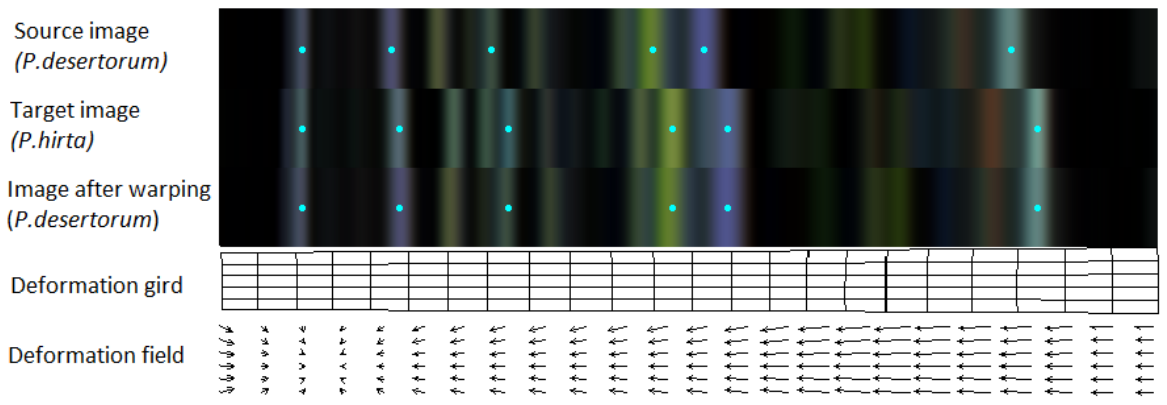

Figure 5. bUnwarpJ warping with landmarks (blue dots in the pictures).
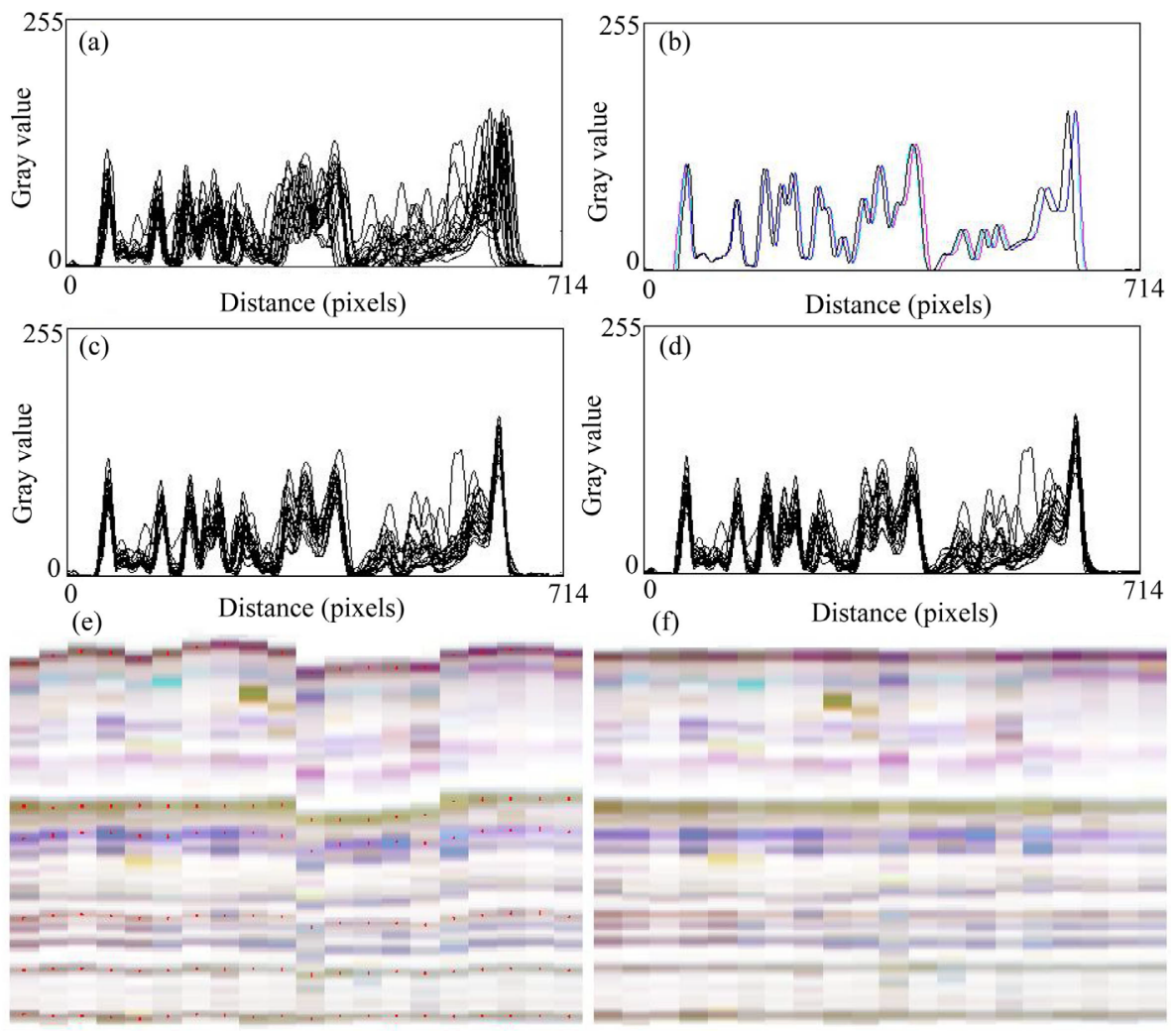

(f)

Distance (pixels)

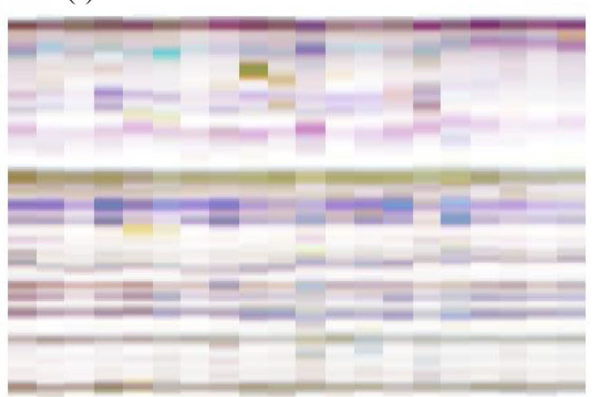

Figure 6. Warping with landmarks (red dots in the pictures). (a) Plot of all subtracks in stack after subtract background; (b) Plot of $P$. desertorum before and after warping (description in text); (c) Plot of all subtracks in stack after subtract background and bUnwarpJ warping; (d) Plot of all subtracks in stack after subtract background and simple, elastic algorithm warping; (e) Invert color subtracks images montage before warping; (f) Invert color subtracks images montage after simple, elastic algorithm warping. 
warping), differences among the Potentilla samples were explored. All the data processing was used to remove the undesired part of the data variation and ensure that all variability of data is related to chemical variability among samples.

The main purpose of our study was to create a fully automatic image processing model that enables quick identification of selected species of the Potentilla genus. Fully processed images of standard samples of the Potentilla species were presented in Figure 7(a). The botanical identity of standard samples was confirmed. Next, three samples which were collected by authors in different time and part of Europe (see Figure 7(b)) were also processed. The final observation of the comparison of each tested sample to the standard samples resulted in highest Pearson correlation coefficient value and the number of standard sample to which the test sample is adjusted (see Table 2).

(a)

P. anserina

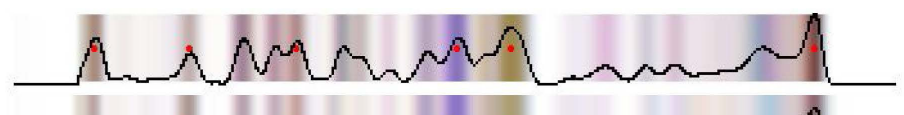

P. hirta

P. fulgens

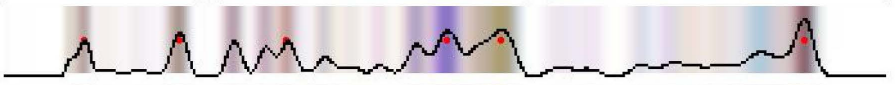

P. crantzii

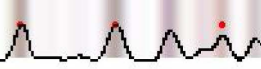

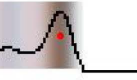

P. crantzii

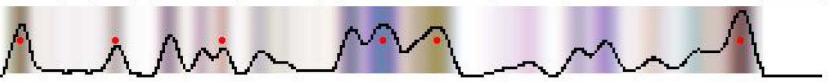

P. grandiffora

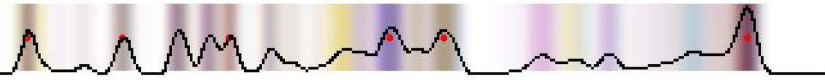

P. montenegrina
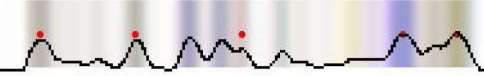

P. recta subsp. obscura

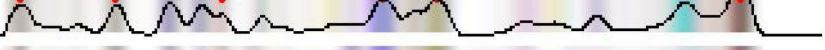

P. recta

P. atrosanguinea

P. argyrophylla

P. erecta

P. desertorum

P. collina

P. megalantha

P. bifurca

P. neumaniana

P. reptans
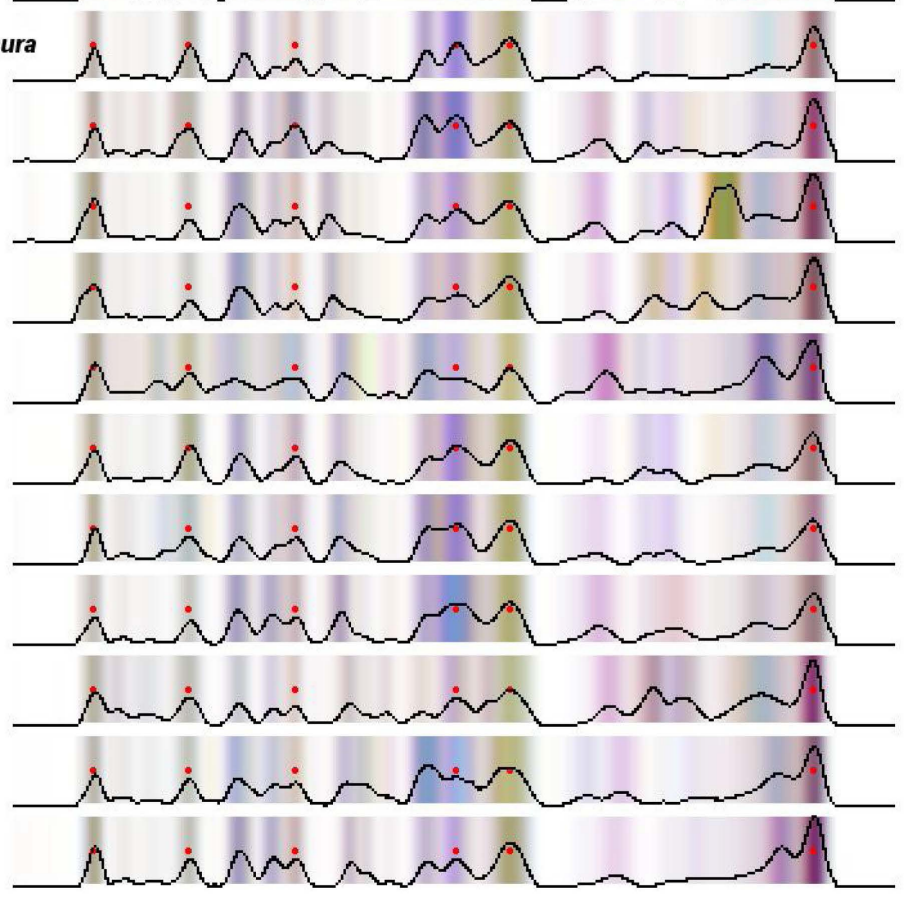

(b)

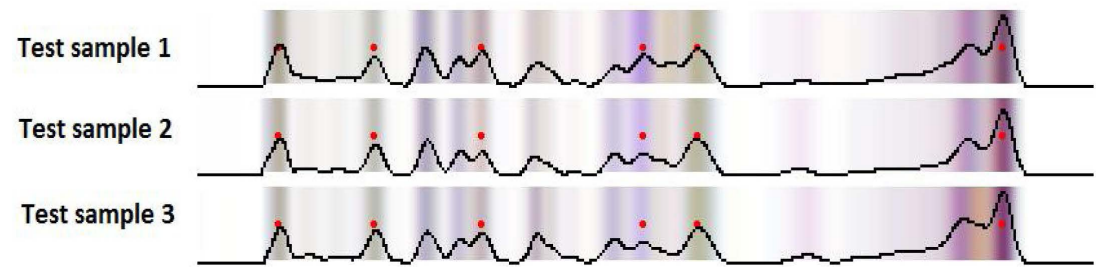

Figure 7. Fully processed invert color images of all Potentilla chromatograms; landmarks (red dots in the pictures). (a) Standard samples; (b) Test samples. 
Table 2. The final result of the comparison of tested sample.

\begin{tabular}{ccc}
\hline \multirow{2}{*}{ Test sample } & \multicolumn{2}{c}{ First match } \\
\cline { 2 - 3 } & Number of best-fit standard sample & $\mathrm{r}$ \\
\hline Bialystok Region, 2008 & 17 & 0.943 \\
France, 2011 & 17 & 0.971 \\
Zamosc Region, 2010 & 17 & 0.933 \\
\hline
\end{tabular}

As it is observed, all test samples are best correlated with the standard sample number 17 (corresponding to $P$. reptans). The most important issue is that test samples were classified as one species, with a correlation coefficient of no less than 0.90 . The correlation coefficient changed in the range from 0.933 to 0.971 for the same species colleting in different places and time. The second best fit standard sample correlation was below 0.9 . Through the use of macros, all image processing (including the removal of noise, subtract background, horizontal equalization, warping) and exploratory analysis (including indicating the number of best-fit standard sample and value of correlation coefficient) from beginning to the end lasted no longer than 30 seconds. To confirm the validity of results, additional botanical analysis of test samples was carried out, thus confirming the accuracy of the obtained results.

Previous observations were also confirmed by the principal component analysis (with auto scaling and mean centering), done on fully processed images. The PCA of processed images showed the grouping of samples of the same species (samples 17, 18, 19 and 20 corresponding to P. reptans collected at different times and places). The first three principal components explain 92\% of total variance. PC2-PC3 loadings plot for all TLC chromatograms is presented in Figure 8.

In the present study, the original and simple method for the separation of closely related five triterpenic acids was presented. The analyzed phytosterols ( $\beta$-sitosterol, stigmasterol) did not interfere. Four triterpenic acids (ursolic/oleanolic acid, pomolic acid and two optical isomers-euscaphic and tormentic acid) were separated without prechromatographic derivatization (see Figure 2(a)). The described method of separating optical isomers is simple, rapid and inexpensive on a silica gel TLC plate, without using expensive impregnated plates, chiral mobile phase or chiral stationary phase. The method is based on different physical properties (unlike enantiomers) and different chemical reactivity of diastereomers. For the separation of oleanolic and ursolic acid, the previously described prechromatographic derivatization method we used [15]. All five triterpene acids can be separated only with prechromatographic derivatization with $1 \%$ iodine solution in chloroform (see Figure 2(b)). It is very important iodine derivatization didn't change retention factors of euscaphic, tormentic and pomolic acid or analyzed sterols.

\section{Conclusions}

The method without prechromatographic derivatization was used to create differential fingerprints. The methodology was validated and may be successfully used to assess the identity of test samples in other laboratories. It seems to be particularly important in this kind of research since fingerprint evaluation method may be used to create a digital database of validated methods with image processing. Such assessment allows identifying different plant species. In our study we proved that the analysis of extracts containing small group of chemical compounds with displaying similar chemical character was sufficient for error-free identification of test samples.

Good results were obtained by the application of r-project iterative algorithm for subtract background and our simple algorithm for warping to image processing of TLC chromatograms for the first time. Fully automated digital data pre-treatment and exploratory analysis of the investigated Potentilla species fingerprints has proved to be fast, simple and effective mechanism for differentiating the investigated plant species. The combination of image processing techniques and statistical evaluation of the collected data proved to be an additional source of interesting conclusions. For this purpose, Pearson's correlation coefficient and the PCA analysis were used.

Fully automated digital data pre-treatment and chemometric analysis has proved to be fast, simple and effective mechanism for differentiating the investigated plant species. This fingerprint evaluation method with image processing may be used to create a digital database for plant identification.

\section{Acknowledgements}

The authors would like to thank Herbapol-Lublin S.A. for the opportunity to perform the research. The authors 


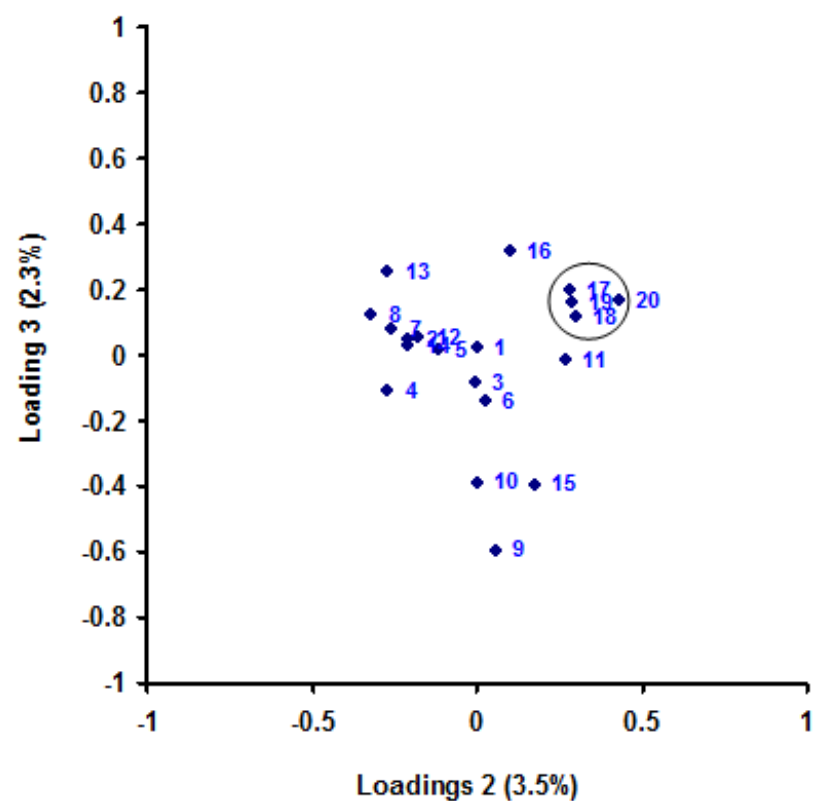

Figure 8. PCA analysis.

also thank the Maria Curie-Sklodowska University Botanical Garden in Lublin for plant material.

\section{References}

[1] EMEA/HMPC/246816/2005 (2005) Guideline on Good Agricultural and Collection Practice (GACP) for Starting Materials of Herbal Origin.

[2] EMA/CPMP/QWP/2820/00 Rev. 2 (2011) Guideline on Specifications: Test Procedures and Acceptance Criteria for Herbal Substances, Herbal Preparations and Herbal Medicinal Products/Traditional Herbal Medicinal Products.

[3] Xie, P., Chen, S., Liang, Y., Wang, X., Tian, R. and Upton, R. (2006) Chromatographic Fingerprint Analysis-A Rational Approach for Quality Assessment of Traditional Chinese Herbal Medicine. Journal of Chromatography A, 1112, 171-180. http://dx.doi.org/10.1016/j.chroma.2005.12.091

[4] Liang, Y.-Z., Xie, P. and Chan, K. (2004) Quality Control of Herbal Medicines. Journal of Chromatography B, 812, 53-70. http://dx.doi.org/10.1016/j.jchromb.2004.08.041

[5] Ma, C.M., Nakamura, N., Hattori, M., Kakuda, H., Qiao, J.C. and Yu, H.L. (2000) Inhibitory Effects on HIV-1 Protease of Constituents from the Wood of Xanthoceras sorbifolia. Journal of Natural Products, 63, 238-242. http://dx.doi.org/10.1021/np9902441

[6] Saraswat, B., Visen, P.K. and Agarwal, D.P. (2000) Ursolic Acid Isolated from Eucalyptus tereticornis Protects against Ethanol Toxicity in Isolated Rat Hepatocytes. Phytotherapy Research, 14, 163-166. http://dx.doi.org/10.1002/(SICI)1099-1573(200005)14:3<163::AID-PTR588>3.0.CO;2-D

[7] Hong, Z., Chen, W., Zhao, J., Wu, Z., Zhou, J., Li, T. and Hu, J. (2010) Hepatoprotective Effects of Rubus aleaefolius Poir. and Identification of Its Active Constituents. Journal of Ethnopharmacology, 129, 267-272. http://dx.doi.org/10.1016/j.jep.2010.03.025

[8] Traore-Keita, F., Gasquet, M., Di Giorgio, C., Ollivier, E., Delmas, F., Keita, A., Doumbo, O., Balansard, G. and Timon-David, P. (2000) Antimalarial Activity of Four Plants Used in Traditional Medicine in Mali. Phytotherapy Research, 14, 45-47. http://dx.doi.org/10.1002/(SICI)1099-1573(200002)14:1<45::AID-PTR544>3.0.CO;2-C

[9] Chattopadhyay, D., Arunachalam, G., Mandal, A.B., Sur, T.K., Mandal, S.C. and Bhattacharya, S.K. (2002) Antimicrobial and Anti-Inflammatory Activity of Folklore: Mallotus peltatus Leaf Extract. Journal of Ethnopharmacology, 82, 229-237. http://dx.doi.org/10.1016/S0378-8741(02)00165-4

[10] Rocha Gda, G., Simões, M., Lúcio, K.A., Oliveira, R.R., Coelho Kaplan, M.A. and Gattass, C.R. (2007) Natural Triterpenoids from Cecropia lyratiloba Are Cytotoxic to Both Sensitive and Multidrug Resistant Leukemia Cell Lines. Bioorganic \& Medicinal Chemistry, 15, 7355-7360. http://dx.doi.org/10.1016/j.bmc.2007.07.020

[11] Li, J., Guo, W.J. and Yang, Q.Y. (2002) Effects of Ursolic Acid and Oleanolic Acid on Human Colon Carcinoma Cell Line HCT15. World Journal of Gastroenterology, 8, 493-495. 
[12] Fogo, A.S., Antonioli, E., Calixto, J.B. and Campos, A.H. (2009) Tormentic Acid Reduces Vascular Smooth Muscle Cell Proliferation and Survival. European Journal of Pharmacology, 615, 50-54. http://dx.doi.org/10.1016/j.ejphar.2009.05.009

[13] Ivorra, M.D., Paya, M. and Villar, A. (1988) Hypoglycemic and Insulin Release Effects of Tormentic Acid: A New Hypoglycemic Natural Product. Planta Medica, 54, 282-286. http://dx.doi.org/10.1055/s-2006-962433

[14] Matysik, G., Wojciak-Kosior, M. and Paduch, R. (2005) The Influence of Calendulae officinalis flos Extracts on Cell Cultures, and the Chromatographic Analysis of Extracts. Journal of Pharmaceutical and Biomedical Analysis, 38, 285-292. http://dx.doi.org/10.1016/j.jpba.2004.12.034

[15] Wójciak-Kosior, M. (2007) Separation and Determination of Closely Related Triterpenic Acids by High Performance Thin-Layer Chromatography after Iodine Derivatization. Journal of Pharmaceutical and Biomedical Analysis, 45, 337 340. http://dx.doi.org/10.1016/j.jpba.2007.05.011

[16] Martelanc, M., Vovk, I. and Simonovska, B. (2009) Separation and Identification of Some Common Isomeric Plant Triterpenoids by Thin-Layer Chromatography and High-Performance Liquid Chromatography. Journal of Chromatography A, 1216, 6662-6670. http://dx.doi.org/10.1016/j.chroma.2009.07.038

[17] Naumoska, K., Simonovska, B., Albreht, A. and Vovk, I. (2013) TLC and TLC-MS Screening of Ursolic, Oleanolic and Betulinic Acids in Plant Extracts. Journal of Planar Chromatography, 26, 125-131. http://dx.doi.org/10.1556/JPC.26.2013.2.4

[18] Savitzky, A. and Golay, M.J.E. (1964) Smoothing and Differentiation of Data by Simplified Least Squares Procedures. Analytical Chemistry, 36, 1627-1639. http://dx.doi.org/10.1021/ac60214a047

[19] Člupek, M., Matějka, P. and Volka, K. (2007) Noise Reduction in Raman Spectra: Finite Impulse Response Filtration versus Savitzky-Golay Smoothing. Journal of Raman Spectroscopy, 38, 1174-1179. http://dx.doi.org/10.1002/jrs.1747

[20] Komsta, Ł. (2009) A Comparative Study on Several Algorithms for Denoising of Thin Layer Densitograms. Analytica Chimica Acta, 641, 52-58. http://dx.doi.org/10.1016/j.aca.2009.03.042

[21] Komsta, Ł. (2009) Suppressing the Charged Coupled Device Noise in Univariate Thin-Layer Videoscans: A Comparison of Several Algorithms. Journal of Chromatography A, 1216, 2548-2553. http://dx.doi.org/10.1016/j.chroma.2009.01.042

[22] Arganda-Carreras, I., Sorzano, C.O.S., Marabini, R., Carazo, J.M., de Solorzano, C.O. and Kybic, J. (2006) Consistent and Elastic Registration of Histological Sections Using Vector-Spline Regularization. Lecture Notes in Computer Science, 4241, 85-95. http://dx.doi.org/10.1007/11889762_8

[23] Komsta, Ł., Cieśla, Ł., Bogucka-Kocka, A., Józefczyk, A., Kryszeń, J. and Waksmundzka-Hajnos, M. (2011) The Start-to-End Chemometric Image Processing of 2D Thin-Layer Videoscans. Journal of Chromatography A, 1218, 2820-2825. http://dx.doi.org/10.1016/j.chroma.2010.12.004

[24] Daszykowski, M. and Walczak, B. (2006) Use and Abuse of Chemometrics in Chromatography. Trends in Analytical Chemistry, 25, 1081-1096. http://dx.doi.org/10.1016/j.trac.2006.09.001 
Scientific Research Publishing (SCIRP) is one of the largest Open Access journal publishers. It is currently publishing more than 200 open access, online, peer-reviewed journals covering a wide range of academic disciplines. SCIRP serves the worldwide academic communities and contributes to the progress and application of science with its publication.

Other selected journals from SCIRP are listed as below. Submit your manuscript to us via either submit@scirp.org or Online Submission Portal.
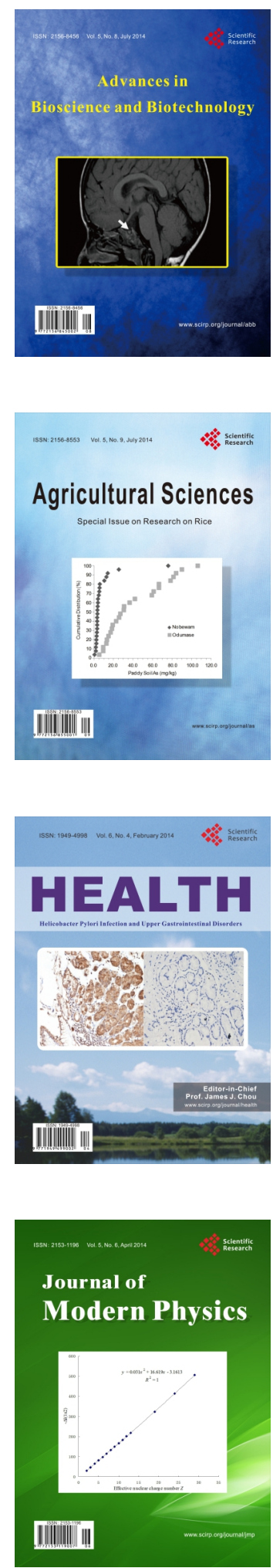
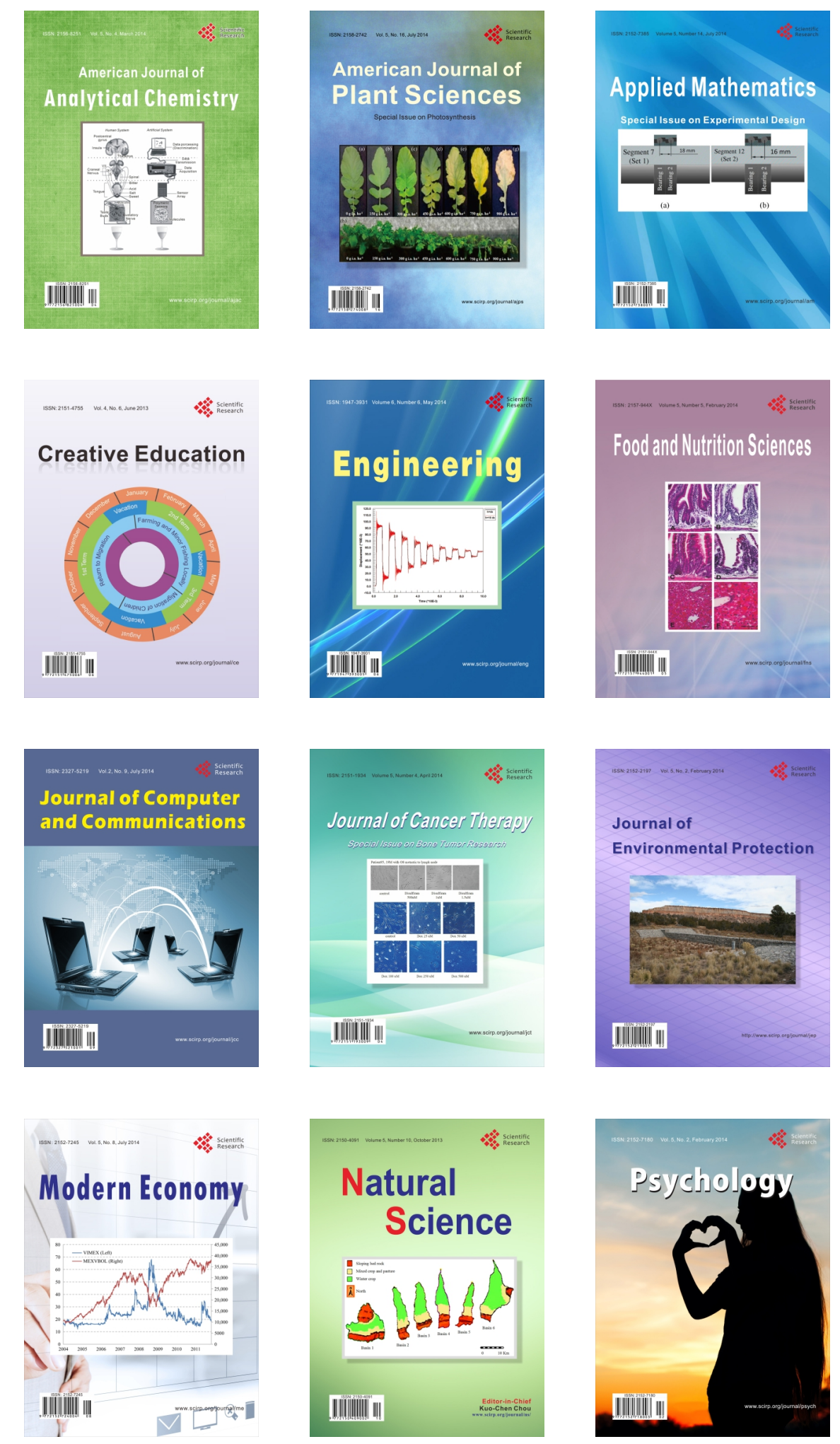